\title{
Analyzing the Friendliness of Exchanges in an Online Software Developer Community
}

\author{
Brendan Cleary, Carlos Gómez, \\ Margaret-Anne Storey \\ University of Victoria \\ Victoria, BC, Canada \\ $\{$ bcleary,cgomez,mstorey\}@uvic.ca
}

\author{
Leif Singer \\ Leibniz Universität Hannover \\ Hannover, Germany \\ leif.singer@inf.uni-hannover.de
}

\author{
Christoph Treude \\ McGill University \\ Montreal, QC, Canada \\ ctreude@gmail.com
}

\begin{abstract}
Many online communities struggle with conflicts e.g. between newcomers and elders - at some point. In July 2012, the Stack Exchange organization attempted to assess the overall "niceness" of the Stack Overflow community by rating the "friendliness" of 7,000 comments made on the site over a 4 year period. We performed a deeper examination of the comment dataset published by Stack Exchange. We find a high degree of comment repetition in the Stack Overflow database and suggest some simple heuristics that may help in automatically identifying unfriendly comments, providing managers of developer communities with simple means that could counter hostility.
\end{abstract}

\section{INTRODUCTION}

Stack Overflow is an increasingly important part of the online resources relied on by millions of software developers [1]-[3]. Like most online communities, newcomer perceptions and future participation can be heavily influenced by their first interactions with the community, especially if they are negative or unfriendly. Being able to automatically classify comment friendliness would allow developer communities like Stack Overflow to preemptively warn users that attempt to post comments which may be received as unfriendly. This could help to reduce the number of unfriendly comments while increasing participation and ultimately the longevity of the community.

To investigate whether Stack Overflow was welcoming or hostile to newcomers, the Stack Exchange organization instigated a campaign in the summer of 2012 to study and promote "niceness" in the Stack Overflow community ${ }^{1}$. Stack Exchange sampled 7,000 of the more than 15 million comments in the Stack Overflow database. Using Mechanical Turk, these comments were manually rated for "friendliness". The Stack Exchange study found that Stack Overflow was becoming a friendlier place, with the percentage of friendly comments increasing over time. However, they also found that first time posters were more likely to receive negative comments $^{2}$.

In this position paper, we perform an analysis of the comment dataset published by Stack Exchange, focusing on the content of those comments and how frequently individual comments are repeated. This is a first step towards building

\footnotetext{
${ }^{1}$ http://blog.stackoverflow.com/?p=11931 (Jul 20, 2012)

${ }^{2}$ https://www.dropbox.com/1/NECSweK1IYKvMBRE (Jul 23, 2012)
}

tools that automatically identify potentially negative comments in developer communities.

\section{Exploring the Stack Exchange Data Set}

Our goal is to determine the viability of automatically classifying the "friendliness" of comments on Stack Overflow and other developer communities. To this end, we are interested in identifying the characteristics of comments that could be used for building a classifier. Specifically we are interested in:

1) Uniqueness - are comments on Stack Overflow unique, or is there repetition that could be utilized by a classification algorithm for comments?

2) Language - are there patterns to vocabulary use that a classification algorithm might use as heuristics?

The Stack Exchange dataset, by providing a pre-classified set of comments, represents a valuable opportunity to address these research questions. To investigate the pervasiveness of friendly and unfriendly comments, we used a simple text matching approach: we attempted to match the 7,000 comments in the published Stack Exchange dataset against the more than 13 million comments contained in the August 2012 Stack Overflow data dump (a snapshot of the Stack Overflow database published by Stack Exchange every 6 months).

We identified 3,561 comments out of the 7,000 that had been published and classified by Stack Exchange. The discrepancy results from comments that were modified or removed from Stack Overflow prior to August 2012.

\section{ANALYSIS \& FINDINGS}

We performed a simple occurrence analysis to identify the number of times each of the 3,561 comments appeared in the August 2012 Stack Overflow data dump and the number of unique users who made the comment. Table I presents the results of this analysis along with a "niceness" classification based on the Stack Exchange study.

The majority of the most frequently repeated comments are classified as "friendly" with only one "unfriendly" comment being highly repeated. In total, we found over 20,000 occurrences of the 3,561 comments. However, only 212 comments were repeated more than once. If the Stack Exchange dataset is representative of Stack Overflow comments, the majority of comments are unique, but a small number are highly repeated. 
TABLE I

TOP 10 COMMENT OCCURRENCES

\begin{tabular}{l|c|c|r} 
Comment & Users & Occurrences & Classification \\
\hline \hline what have you tried? & 1,472 & 3,570 & FRIENDLY \\
\hline what have you tried so far? & 981 & 1,834 & FRIENDLY \\
\hline is this homework? & 939 & 1,530 & UNFRIENDLY \\
\hline you're welcome! & 603 & 1,001 & FRIENDLY \\
\hline what is your question? & 605 & 892 & FRIENDLY \\
\hline what's the question? & 364 & 477 & FRIENDLY \\
\hline what is the error? & 373 & 433 & FRIENDLY \\
\hline what error do you get? & 300 & 382 & FRIENDLY \\
\hline thanks very much! & 263 & 323 & FRIENDLY \\
\hline what error are you getting? & 277 & 312 & FRIENDLY
\end{tabular}

TABLE II

COMMENT REPEATERS

\begin{tabular}{l|c|c} 
Comment & Users & Occurrences \\
\hline \hline please learn java naming ... & 1 & 150 \\
\hline needs ‘homework' tag? & 1 & 73 \\
\hline you can get help from facebook ... & 1 & 40 \\
\hline i am doing something similar ... & 1 & 26 \\
\hline can you answer your own ... & 1 & 24
\end{tabular}

Another notable finding were comments that occurred frequently, but were made by only a small number of users. For example, Table II lists a selection from the 100 most frequently occurring comments that each originated from a single user (comments have been abbreviated). Table III shows the top 10 most frequently occurring "unfriendly" comments. Notably, the "homework" keyword is not only present in the most repeated "unfriendly" comment, but is also present in half of the top 10 "unfriendly" comments. This constrained vocabulary presents a simple heuristic that could be used to flag "unfriendly" comments.

Table IV shows the most frequently occurring ambiguously classified comments. In comparison to the most frequently occurring "friendly" or "unfriendly" comments, ambiguous comments tend to be repeated far less often and appear to have a richer vocabulary.

\section{LiMITATIONS}

As we examined only comments that remained in the Stack Overflow database in August 2012, there is a possibility that extremely negative comments captured in the Stack Exchange study might have been deleted before this date. Because the

TABLE III

TOP 10 OCCURRING UNFRIENDLY COMMENTS

\begin{tabular}{l|c|c} 
Comment & Users & Occurrences \\
\hline \hline is this homework? & 939 & 1,530 \\
\hline and the question is? & 148 & 180 \\
\hline belongs on superuser.com & 29 & 108 \\
\hline is this a homework? & 56 & 74 \\
\hline needs 'homework' tag? & 1 & 73 \\
\hline smells like homework. & 50 & 69 \\
\hline what are you asking? & 33 & 65 \\
\hline not programming related & 30 & 54 \\
\hline so what is the question? & 50 & 50 \\
\hline homework question? & 45 & 48
\end{tabular}

TABLE IV

Top 10 OCCURRING AmBIguOUS COMMEnTs

\begin{tabular}{l|c|c} 
Comment & Users & Occurrences \\
\hline \hline why the downvotes? & 85 & 89 \\
\hline $\begin{array}{l}\text { this question is not a good fit to our } \\
\text { q\&a format .. }\end{array}$ & 9 & 21 \\
\hline belongs on superuser & 17 & 20 \\
\hline what flavor of sql? on program- & 6 & 11 \\
\hline $\begin{array}{l}\text { belongs } \\
\text { mers.stackexchange.com }\end{array}$ & 1 & 8 \\
\hline needs to be cw? & 1 & 7 \\
\hline $\begin{array}{l}\text { your question is better suited for server- } \\
\text { fault.com }\end{array}$ & 4 & 7 \\
\hline why is this off topic? & 1 & 3 \\
\hline community wiki ...? & 3 & 3
\end{tabular}

Stack Exchange dataset contained comments sampled at specific times over four years, it is possible that some frequently occurring comments were not captured in that dataset, and therefore would not be considered in our analysis. Finally, the comment classifications provided in the Stack Exchange dataset were collected using Mechanical Turk: participants were not explicitly trained in comment classification and did not have access to the context in which a comment appeared.

\section{CONClusions \& Future Work}

Using data published by Stack Exchange that rated the "friendliness" of Stack Overflow comments, we analyzed how frequently those comments were repeated on Stack Overflow and the vocabulary used in different types of comments. We found that the most frequently occurring "unfriendly" comments use a constrained vocabulary and that some very frequently repeated comments are created by small numbers of users. This qualitative analysis adds further insights to the quantitative analysis conducted by Stack Exchange so far. Our findings suggest possible features that could be implemented on Stack Overflow and other developer communities that would warn users that are about to post a comment that might be received as negative. In future work, we will investigate the automatic classification of comments and posts.

\section{ACKNOWLEDGMENTS}

We thank Stack Exchange for publishing their data. The Stack Overflow data dump is licensed under a Creative Commons license (CC-BY-SA 3.0).

\section{REFERENCES}

[1] B. Dagenais and M. Robillard, "Creating and evolving developer documentation: understanding the decisions of open source contributors," in Proceedings of the eighteenth ACM SIGSOFT international symposium on Foundations of software engineering. ACM, 2010, pp. 127-136.

[2] S. M. Nasehi, J. Sillito, F. Maurer, and C. Burns, "What makes a good code example?: A study of programming q\&a in stackoverflow," in Software Maintenance (ICSM), 2012 28th IEEE International Conference on, sept. 2012, pp. 25-34.

[3] C. Parnin and C. Treude, "Measuring api documentation on the web," in Proceedings of the 2nd International Workshop on Web 2.0 for Software Engineering, ser. Web2SE '11. New York, NY, USA: ACM, 2011, pp. 25-30. [Online]. Available: http://doi.acm.org/10.1145/1984701.1984706 